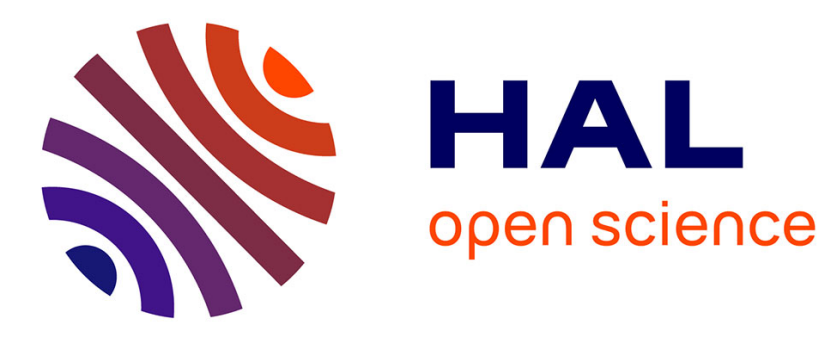

\title{
Simulation of Astrocytic Calcium Dynamics in Lattice Light Sheet Microscopy Images
}

Anaïs Badoual, Misa Arizono, Audrey Denizot, Mathieu Ducros, Hugues Berry, U Valentin Nägerl, Charles Kervrann

\section{- To cite this version:}

Anaïs Badoual, Misa Arizono, Audrey Denizot, Mathieu Ducros, Hugues Berry, et al.. Simulation of Astrocytic Calcium Dynamics in Lattice Light Sheet Microscopy Images. ISBI 2021

- IEEE International Symposium on Biomedical Imaging, Apr 2021, Nice, France. pp.1-5, 10.1109/ISBI48211.2021.9433984 . hal-03106797

\section{HAL Id: hal-03106797 \\ https://hal.inria.fr/hal-03106797}

Submitted on 12 Jan 2021

HAL is a multi-disciplinary open access archive for the deposit and dissemination of scientific research documents, whether they are published or not. The documents may come from teaching and research institutions in France or abroad, or from public or private research centers.
L'archive ouverte pluridisciplinaire HAL, est destinée au dépôt et à la diffusion de documents scientifiques de niveau recherche, publiés ou non, émanant des établissements d'enseignement et de recherche français ou étrangers, des laboratoires publics ou privés. 


\title{
SIMULATION OF ASTROCYTIC CALCIUM DYNAMICS IN LATTICE LIGHT SHEET MICROSCOPY IMAGES
}

\author{
Anaïs Badoual $^{1}$, Misa Arizono ${ }^{2}$, Audrey Denizot ${ }^{3}$, Mathieu Ducros ${ }^{4}$, Hugues Berry $^{5,6}$, \\ U. Valentin Nägerl ${ }^{2}$, Charles Kervrann ${ }^{1}$ \\ ${ }^{1}$ Inria, Centre de Recherche Bretagne Atlantique, Serpico Team, Rennes, France \\ ${ }^{2}$ Interdisciplinary Institute for Neuroscience, UMR5297 CNRS, Bordeaux, France \\ ${ }^{3}$ Computational Neuroscience Unit, Okinawa Institute of Science and Technology, Onna, Japan \\ ${ }^{4}$ Bordeaux Imaging Center, UMS3420 CNRS, US04 INSERM, Bordeaux, France \\ ${ }^{5}$ Inria, Centre de Recherche Rhone-Alpes, Beagle Team, F-69603, Villeurbanne, France \\ ${ }^{6}$ Univ Lyon, LIRIS, UMR5205 CNRS, F-69621, Villeurbanne, France
}

\begin{abstract}
Astrocytes regulate neuronal information processing through a variety of spatio-temporal calcium signals. Recent advances in calcium imaging have started to shine light on astrocytic activity, but the complexity and size of the recorded data strongly call for more advanced computational analysis tools. Their development is currently hindered by the lack of reliable, labeled annotations that are essential for the evaluation of algorithms and the training of learning-based methods. To solve this labeling problem, we have designed a generator of 2D/3D lattice light sheet microscopy (LLSM) sequences which realistically depict the calcium dynamics of astrocytes. By closely modeling calcium kinetics in real astrocytic ramifications, the generated datasets open the door for the deployment of convolutional neural networks in LLSM.
\end{abstract}

Index Terms - Astrocytes, calcium signals, image analysis, lattice light sheet microscopy, synthetic training datasets.

\section{INTRODUCTION}

Context. Astrocytes are glial cells in the central nervous system that recently emerged as key partners of neurons for the processing of information [1]. Astrocytes sense synaptic activity through the detection of neurotransmitters, and respond to it by varying their own cytosolic calcium concentration. This then triggers the release of gliotransmitters that further regulate neuronal communication [2].

Astrocytic calcium signals are involved in many key brain functions (e.g., memory and learning [1]), and their alterations can lead to brain diseases [1]. These signals also exhibit an important spatiotemporal diversity $[3,4]$, and it is still unknown whether this variability relates to their role in distinct neurobiological functions. Not surprisingly, decoding this "calcium code" is a leading topic in neuroscience [5]. The challenge is that the recording of astrocytic calcium activity is an intricate task that requires high resolution in both space and time. Indeed, most signals occur in fine astrocyte ramifications $(50-200 \mathrm{~nm}[6])$, called processes, and last between milliseconds to tens of seconds [3].

Recent efforts to overcome this imaging challenge include the use of 3D super-resolution STED microscopy [7] and computational

This work was partially supported by the French National Research Agency (France-BioImaging infrastructure-ANR-10-INBS-04).

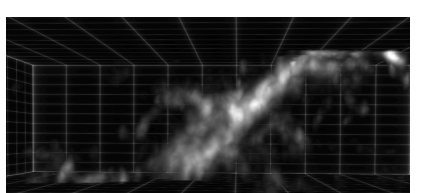

(a) $t=16.5 \mathrm{~s}$.

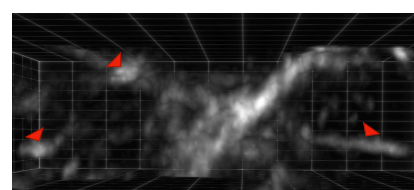

(b) $t=43.0 \mathrm{~s}$.
Fig. 1. 3D views of astrocytic calcium signals in real $3 D+t i m e$ LLSM images. Red triangles: changes in calcium signals. Voxelsize: $0.1025 \times 0.1025 \times 0.1066 \mu \mathrm{m}^{3}$. Acquisition frequency: $2 \mathrm{~Hz}$. Source: M. Arizono, M. Ducros, U. V. Nägerl, Interdisciplinary Institute for Neuroscience, Bordeaux.

modeling $[8,9]$. The trade-off between temporal and spatial resolution in microscopy techniques has mostly restricted the quantification of astrocytic calcium signals in 2D+time images. Unfortunately, key information can be lost by ignoring the third dimension, such as the number of calcium signals orchestrated in a single astrocyte and the location from which they originated [4].

The recent emergence of lattice light sheet microscopy (LLSM) $[10,11]$ now enables a high spatio-temporal resolution with low phototoxicity. Together with genetically encoded calcium indicators (GECIs) [12], this finally provides a refined 3D imaging of the dynamical behavior of calcium signals in astrocytes (see Fig. 1).

Need for Simulation Tools. Analysing astrocytic calcium signals in sequences of LLSM images is challenging due to the complex nature of these signals. For one, they are characterized by various frequencies, durations, and signal-to-noise ratios (SNR). Second, they can be localized within microdomains or propagate within the astrocyte in regions of various sizes [3]. The community is currently lacking of image analysis tools to detect, segment and quantify these signals in LLSM images. The few existing are mostly dedicated to 2D+time fluorescence microscopy $[13,14,15,16]$.

The development of these tools is hindered by the lack of large reliable labeled data needed for evaluation and training. Manual annotation of LLSM sequences is close to impossible, especially in $3 \mathrm{D}+$ time images for which the complex visualization may lead to a significant intra- and inter-experimenter variability. Hence, a common and promising practice is to use realistic synthetic datasets with known ground-truth to train and quantitatively assess the performance of analysis software [17]. 
Generating such ground-truth images of astrocytic calcium activity is as a critical task. In previous works $[15,16]$, some simplifications were made: only one type of calcium signal was represented at a time, the background contained none or only small parts of astrocytes, or the third dimension was omitted. Moreover, the corresponding implementations were not always provided. To the best of our knowledge, there is currently no free available simulation tool to generate realistic sequences of $2 \mathrm{D}$ and $3 \mathrm{D}$ images depicting calcium dynamics in the sponge-like network of astrocytic processes.

Contributions. We present a framework to simulate realistic astrocytic calcium signals in sequences of 2D and 3D LLSM images. To reproduce the spatio-temporal diversity of calcium signals, we adapt at the macroscale the kinetic model described in [18] which represents calcium dynamics at the nanoscale. For the background, we use real astrocytic processes and we recreate experimental conditions of LLSM acquisitions (e.g., noise and blurring). This provides realistic datasets that can be used for both evaluating algorithm performance and training convolutional neural networks. We are hopeful that this tool will allow the development of learning-based analysis tools to quantify astrocytic calcium signals in $3 \mathrm{D}+$ time images.

\section{KINETIC MODEL}

Several kinetic models of calcium dynamics have been developed $[8,9]$. In this section, we describe the one we use for our simulations, a simplified version of the model developed by Denizot et al. [18].

\subsection{Calcium Fluxes in the Cytosol}

Calcium signals correspond to a transient increase of calcium concentration in the cytosol. They result from a flux of calcium into the cytosol, arising from the opening of calcium channels. The channel responsible for the majority of calcium signals in astrocytes is the inositol 1,4,5-triphosphate receptor $\left(\mathrm{IP}_{3} \mathrm{R}\right)$ [3]. It is located at the surface of the endoplasmic reticulum (ER), an internal store characterized by a calcium concentration that is 3 orders of magnitude higher than the cytosol (see Fig. 2) [19].

$\mathrm{IP}_{3} \mathrm{Rs}$ can form clusters on the membrane of the ER [20]. The probability for an $\mathrm{IP}_{3} \mathrm{R}$ to be in the open state has a bell-shaped dependence to cytosolic calcium concentration $\left[\mathrm{Ca}^{2+}\right]$ [21]. This means that a small increase in the local $\left[\mathrm{Ca}^{2+}\right]$, resulting from a stimulus for example, increases the open probability of $\mathrm{IP}_{3} \mathrm{Rs}$. This is called the calcium-induced-calcium release (CICR) mechanism. However, as soon as $\left[\mathrm{Ca}^{2+}\right]$ raises above a certain threshold, this probability decreases significantly. The state of an $\mathrm{IP}_{3} \mathrm{R}$ does not change instantly. Hence, we consider that an $\mathrm{IP}_{3} \mathrm{R}$ stays $\tau_{o}$ and $\tau_{c}$ seconds in the open and closed states, respectively. An open $\mathrm{IP}_{3} \mathrm{R}$ injects calcium into the cytosol at a constant rate $\mu . \mathrm{Ca}^{2+}$ outflow from the cytosol towards the extracellular space or the ER are combined through the decay rate $\alpha$ (see Fig. 2) [18].

\subsection{Calcium Diffusion}

Calcium ions diffuse in the cytosol through Brownian motion [18]. The position $\mathbf{r}$ of each $C a^{2+}$ is given by $\mathbf{r}\left(t+\Delta t_{\mathrm{e}}\right)=\mathbf{r}(t)+$ $\sqrt{2 D \Delta t_{\mathrm{e}}} \xi$, where $D$ is the diffusion coefficient of a calcium ion, $\xi$ is a vector of i.i.d. Gaussian-distributed random numbers with zero mean and unit variance, and $\Delta t_{\mathrm{e}}$ is the time interval between two updated positions of the calcium ion.

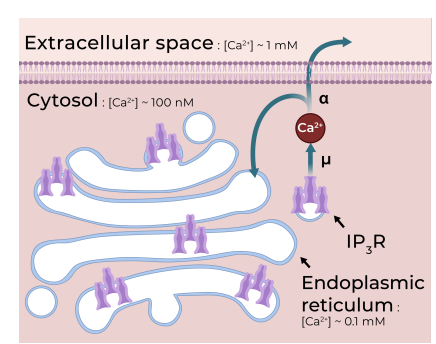

(a) Calcium fluxes.

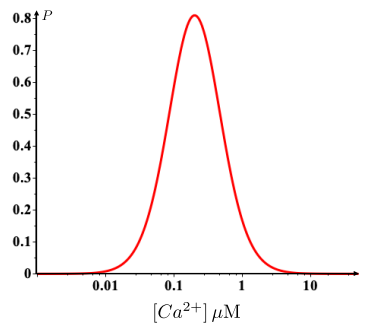

(b) Open probability of an $\mathrm{IP}_{3} \mathrm{R}$.
Fig. 2. Scheme of the kinetic model used for our simulation. (a) Calcium enters the cytosol through open $\mathrm{IP}_{3} \mathrm{Rs}$ at a rate $\mu$ and leaves to the extracellular space or the ER at a rate $\alpha$. (b) The probability of an $\mathrm{IP}_{3} \mathrm{R}$ to be in the open state is modeled by a bell-shape function that we approximate by $P\left(\left[\mathrm{Ca}^{2+}\right]\right)=\left(a \frac{0.2\left[\mathrm{Ca}^{2+}\right]}{\left(\left[\mathrm{Ca}^{2+}\right]+0.2\right)^{2}}\right)^{2.7}[21]$. Here $a=3.7$.

\subsection{Spatio-Temporal Diversity of Calcium Signals}

Calcium signals are usually classified in three different types: blips, puffs and waves [22] (see Fig. 4 and 6). Calcium blips are weak and brief signals that result from the opening of a single $\mathrm{IP}_{3} \mathrm{R}$. Calcium puffs are peaks of higher amplitude and duration. They result from the concerted opening of a few $\mathrm{IP}_{3} \mathrm{Rs}$, potentially organized into a cluster. Finally, calcium waves correspond to the propagation of calcium puffs from one cluster to neighboring clusters via the CICR mechanism [18]. There is a diversity of calcium waves. Depending on the spatial organization of the clusters, waves propagate over different distances, move toward or from the astrocyte cell body, and the propagation can grow or move (see Fig. 4 (b) and (c), respectively). All these calcium events occur in regions of various sizes and may overlap in space and time with other calcium signals.

\subsection{Simplifications}

The kinetic model presented in [18] describes two additional mechanisms that are essential at the nanoscale. First, calcium ions can also enter the cytosol from the extracellular space or the ER, independently from $\mathrm{IP}_{3} \mathrm{Rs}$. This flux has a constant rate that is negligible compared to $\alpha$ and $\mu$, yet essential to maintain a basal $\left[\mathrm{Ca}^{2+}\right]$ in the cytosol. Second, $\mathrm{IP}_{3} \mathrm{Rs}$ are co-activated by $\mathrm{Ca}^{2+}$ and a molecule called $\mathrm{IP}_{3}$. In our kinetic model, we omit these two mechanisms. We implicitly account for the influence of the $\mathrm{IP}_{3}$ concentration by varying both the amplitude of the open probability (see Fig. 2 (b)) and the local $\left[\mathrm{Ca}^{2+}\right]$ increase induced by a stimulus [23]. The basal $\left[\mathrm{Ca}^{2+}\right]$ is implicitly simulated by adding a Gaussian noise of nonzero mean during image synthesis (see Section 3.5).

\section{SIMULATION FRAMEWORK}

In this section, we describe the integration of the kinetic model described in Section 2 into our framework to generate sequences of LLSM 2D/3D images that depict dynamical astrocytic calcium signals. For comprehensiveness, we focus here on our framework for 3D+time images.

\subsection{Notations}

We denote by $t$ a continuous parameter in $\mathbb{R}^{+}$and by $\mathbf{x}=(x, y, z)$ a coordinate vector. We simulate astrocytic activity during $T$ seconds 
through a sequence $f: \Omega \times[0, T] \rightarrow \mathbb{R}$ with a temporal resolution of $\Delta t s$. The symbol $\Omega \subset \mathbb{R}^{3}$ denotes the domain of definition of each volume of $f$. We denote by $f_{\mathrm{e}}: \Omega \times[0, T] \rightarrow \mathbb{R}$ the sequence that simulates a single calcium event of $f$ at higher temporal resolution $\Delta t_{\mathrm{e}}$, with $\Delta t_{\mathrm{e}}<\Delta t$. The symbols $\boldsymbol{\nabla}$ and $\mathbb{1}_{\mathcal{B}}$ denote the gradient operator and the indicator function of a subset $\mathcal{B}$, respectively.

\subsection{Spatial Mask of Astrocytes}

We build a 3D binary mask $I: \Omega \rightarrow\{0,1\}$ of astrocytic processes from a real sequence of LLSM volumes that provides regions of interest in which simulated calcium signals occur. We first apply an Hessian-based multiscale filter [24] on the maximum intensity projection of the real sequence to enhance the astrocytic processes present in the volume. Then, we perform a threshold on the intensity value. Finally, we apply morphology closing and opening operators to fill the holes and remove noise in the mask. From $I$, we define $\mathcal{A}=\{\mathbf{x} \in \Omega \mid I(\mathbf{x})=1\}$ and $\mathcal{E}=\{\mathbf{x} \in \Omega \mid\|\nabla I(\mathbf{x})\| \neq 0\}$ the sets of points belonging to astrocytes and their edges, respectively.

\subsection{Simulation of Calcium Blips and Puffs}

We consider a stimulus at time $t_{0}$ and location $\mathbf{x}_{c}$ of an astrocyte that triggers a $\gamma$ variation of $\left[\mathrm{Ca}^{2+}\right]$ over $0.1 \mathrm{~s}$. We model it by a Gaussian function $g$ centered in $\mathbf{x}_{c}$ with standard deviation $\sigma$, i.e.,

$$
\forall \mathbf{x} \in \Omega, g(\mathbf{x})= \begin{cases}\gamma \mathrm{e}^{-\left(\frac{\left\|\mathbf{x}-\mathbf{x}_{c}\right\|^{2}}{2 \sigma^{2}}\right)} & \text { if } \mathbf{x} \in \mathcal{A}, \\ 0 & \text { otherwise. }\end{cases}
$$

To simulate the calcium signal induced by an $\mathrm{IP}_{3} \mathrm{R}$ cluster in response to this stimulus via CICR, we implement the calcium fluxes and $\mathrm{Ca}^{2+}$ diffusion described in Sections 2.1 and 2.2, respectively. To represent Brownian motion at the macroscopic scale, isotropic diffusion has to be simulated and maintained inside astrocytic processes. To do this, we use the anisotropic diffusion developed by Perona-Malik [25] that stops isotropic diffusion at edges. The partial differential equation describing the diffusion process is given by

$$
\left\{\begin{array}{l}
\frac{\partial f_{\mathrm{e}}(\mathbf{x}, t)}{\partial t}=\operatorname{div}\left(c\left(\left\|\boldsymbol{\nabla} f_{\mathrm{e}}(\mathbf{x}, t)\right\|\right) \boldsymbol{\nabla} f_{\mathrm{e}}(\mathbf{x}, t)\right) \\
f_{\mathrm{e}}\left(\mathbf{x}, t_{0}\right)=g(\mathbf{x})+\beta \mathbb{1}_{\mathcal{E}}(\mathbf{x}),
\end{array}\right.
$$

where $g$ is defined by (1) and $\operatorname{div}(\cdot)$ is the divergence operator. The term $\beta \mathbb{1}_{\mathcal{E}}(\mathbf{x})$ corresponds to the edges of astrocytic processes added with maximum intensity $\beta$ in the volume. The function $c$ is the conductivity function chosen as the Gaussian kernel defined by $c(u)=\exp \left(-\left(\frac{u}{\kappa}\right)^{2}\right)$, where $u \in \mathbb{R}$ and $\kappa$ is a parameter that controls the sensitivity to edges. By adding the calcium fluxes to $\mathrm{Ca}^{2+}$ diffusion, the cytosolic calcium concentration at location $\mathrm{x} \in \Omega$ and at time $t+\Delta t_{\mathrm{e}}$ is given by

$$
\begin{aligned}
f_{\mathrm{e}}\left(\mathbf{x}, t+\Delta t_{\mathrm{e}}\right)= & f_{\mathrm{e}}(\mathbf{x}, t)+\Delta t_{\mathrm{e}} \frac{\partial f_{\mathrm{e}}(\mathbf{x}, t)}{\partial t} \\
& +\Delta t_{\mathrm{e}}\left(\sum_{n=1}^{N_{c}} \mu \mathbb{1}_{\mathcal{O}_{t}}(n)-\alpha\right),
\end{aligned}
$$

where $N_{c}$ is the number of $\operatorname{IP}_{3} \mathrm{R}$ in the cluster, and $\mathcal{O}_{t}$ is the set of open $\mathrm{IP}_{3} \mathrm{Rs}$ at time $t$. To determine $\mathcal{O}_{t}$, we approximate the open probability by the bell-shape function given in Fig. 2 (b) and we wait for $\tau_{o} \mathrm{~S}\left(\tau_{c} \mathrm{~s}\right.$, respectively) before updating the state of an open (closed, respectively) $\mathrm{IP}_{3} \mathrm{R}$. We simulate a calcium puff by iteratively computing Eq. (3) over time. For calcium blips, we simply take $N_{c}=1$.

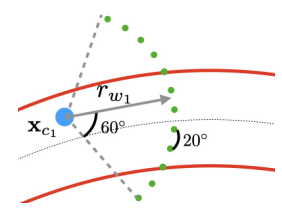

(a) Determination $\mathbf{x}_{c_{2}}$.

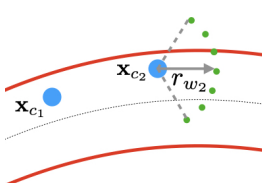

of (b) Determination $\mathbf{x}_{c_{3}}$

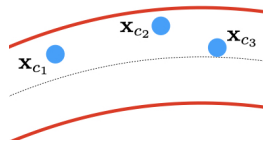

(c) The three calcium puff sites of the wave.
Fig. 3. 2D illustration of the pipeline to find the puff sites $\left\{\mathbf{x}_{c_{n}}\right\}_{n \in\{1,2,3\}}$ of a calcium wave for $N_{w}=3$.

\subsection{Simulation of Calcium Waves}

We consider that a calcium wave is a succession of calcium puffs induced by $N_{w}$ clusters. Each cluster is defined by its center location $\mathbf{x}_{c_{n}}$ and its distance $r_{w_{n}}$ to its neighboring cluster. Note that the $r_{w_{n}}$ are not identical (see Table 1 for their values). The location $\mathbf{x}_{c_{1}}$ of the first cluster is chosen randomly in $\mathcal{A}$. The center $\mathbf{x}_{c_{2}}$ is found as follows (see Fig. 3): we compute the centerline of the binary mask $I$ in the vicinity of $\mathbf{x}_{c_{1}}$ using a skeletonization method to have the local direction of the astrocytic process. Then, we place points uniformly $\left(20^{\circ}\right.$ spacing) on a sphere segment defined by an angle of $60^{\circ}$ with respect to the local direction of the astrocytic process and a radius equal to $r_{w_{1}}$. Finally, we randomly choose one of these points as $\mathbf{x}_{c_{2}}$ with $\mathbf{x}_{c_{2}} \in \mathcal{A}$. We reiterate this approach to find the center of each cluster. The calcium puff triggered by each cluster is simulated using the approach described in Section 3.3. The triggered moments $\left\{t_{0_{n}}\right\}_{n \in\left\{1, \ldots, N_{w}\right\}}$ are chosen randomly such that $t_{0_{1}}<t_{0_{2}}<\ldots<t_{0_{N_{w}}}<T$ and $t_{0_{n}}-t_{0_{n-1}}<\tau_{w} s$.

\subsection{D LLSM Image Synthesis}

We consider astrocytic activity with $N_{s}$ stimuli that trigger the calcium signals $\left\{f_{\mathrm{e}_{n}}\right\}_{n \in\left\{1, \ldots, N_{s}\right\}}$. Among these $N_{s}$ signals, we generate $5 \%$ of blips, $60 \%$ of puffs and $35 \%$ of waves. The final sequence $f$ is obtained by:

1. combining the calcium signals $\left\{f_{\mathrm{e}_{n}}\right\}_{n \in\left\{1, \ldots, N_{s}\right\}}$

2. removing the edges of astrocytes added in Eq. (2),

3 . adding a real astrocytic background $I_{b}$, which is extracted from the real sequence used in Section 3.2 at a time when no or few calcium events occur,

4. adding both a Poisson and a Gaussian noise to simulate the two main sources of noise in LLSM acquisition.

To summarize, $f$ is given by, $\forall \mathbf{x} \in \Omega$,

$f(\mathbf{x}, t)=\sum_{n=1}^{N_{s}}\left(f_{\mathrm{e}_{n}}(\mathbf{x}, t)-\beta \mathbb{1}_{\mathcal{E}}(\mathbf{x})\right)+I_{b}(\mathbf{x})+g_{0} \mathfrak{N}(\mathbf{x})+\epsilon(\mathbf{x})$,

where $\epsilon(\mathbf{x}) \sim \mathcal{N}\left(m_{\epsilon}, \sigma_{\epsilon}\right)^{2}$ is the Gaussian noise representing dark current, $g_{0}$ is the gain of the electronic system, and $\mathfrak{N}(\mathbf{x})$ is the number of photo-electrons at $\mathbf{x}$ assumed to be Poisson distributed with unknown mean $\theta(\mathbf{x})$. We approximate the blurring effect due to the point spread function of the LLSM by a Gaussian filtering whose standard deviation depends on the resolution of the image. Finally, we discretize and sample $f$ at the acquisition frequency such that $f[n]=f(n \Delta t), n=0, \ldots,\lfloor T / \Delta t\rfloor$.

\section{EXPERIMENTAL RESULTS}

We have implemented the framework described in this paper as a plugin within the open source image-processing package Fiji [26]. 
Table 1. Parameters of the kinetic model. [,]: randomly (rdm) chosen in the interval.

\begin{tabular}{|c|c||c|c|}
\hline Parameter & Value & Parameter & Value \\
\hline$\Delta t_{\mathrm{e}}$ & $0.01 s$ & $\gamma$ & {$[0.1,0.3] \mu \mathrm{M}$} \\
$N_{c}$ & 3 & $\sigma$ & {$[0.1,0.5] \mu \mathrm{m}$} \\
$\mathbf{x}_{c}$ & $\operatorname{rdm} \operatorname{in} \mathcal{A}$ & $t_{0}$ & {$[0, T] s$} \\
$\mu$ & $1 \mu \mathrm{M} \cdot \mathrm{s}^{-1}$ & $N_{w}$ & {$[3,10]$} \\
$\tau_{o}$ & $0.01 s$ & $r_{w}$ & {$[0.5,3] \mu \mathrm{m}$} \\
$\tau_{c}$ & $0.2 s$ & $\tau_{w}$ & $1 s$ \\
$\alpha$ & $0.5 \mu \mathrm{M} \cdot s^{-1}$ & $\kappa$ & 40 \\
$a$ & {$[3.5,3.7]$} & & \\
\hline
\end{tabular}

\subsection{Parameter Settings}

Our simulation framework generates a large diversity of datasets by changing only few parameters: the spatial domain $\Omega$ of the image, the acquisition time $T$, the temporal sampling $\Delta t$ (which acts as a temporal window under which calcium signals cannot be observed), the number of stimuli $N_{\mathrm{s}}$ (which controls the level of astrocytic activity), and the amplitude of noise $\sigma_{\epsilon}$. A set of 2D and 3D real backgrounds $I_{b}$ is provided to the user. They are obtained from real data acquired with the LLS microscope developed by Ducros et al. [11] with lateral and axial resolutions of $273 \mathrm{~nm}$ and $558 \mathrm{~nm}$, respectively. The values of the kinetic model parameters (see Table 1) are chosen with respect to the experimental results reported in $[18,23]$.

\subsection{Evaluation}

A quantitative comparison with real LLSM data is not possible. As a start, a visual inspection of our synthetic data by a panel of biologists confirms that they are convincingly realistic both in terms of calcium dynamics and of noisy astrocytic background. A visual comparison to real data (see Fig. 4, 5 and 6) shows that our synthetic data represent well the spatio-temporal diversity of calcium signals. More precisely, puffs and waves of different durations (from $m s$ to $s$ ) and amplitudes, occurring at locations of various sizes and overlapping in space and time can be observed. Note that we cannot compare our simulated blips to experimental data, as the resolution of the latter is too low to determine the exact number of open $\mathrm{IP}_{3} \mathrm{Rs}$. The traces presented in Fig. 6 were obtained by extracting the temporal intensity profile of the epicenters of the calcium puffs. For a better visualization of our 2D and 3D datasets, we provide videos of live synthetic astrocytic activity at https://team.inria.fr/serpico/astrocytic-calcium-dynamics/. The average time to generate a $2 \mathrm{D}$ sequence $(200$ frames of size $170 \times 512$ pixels) with $N_{s}=100$ calcium signals is about one minute on a $2.8 \mathrm{GHz} \mathrm{CPU}$ with $16 \mathrm{~GB}$ RAM. For a 3D sequence, the time is proportional to the number of z-slices ( $48 \mathrm{z}$-slices for $\Delta t=0.5 \mathrm{~s}$ ).

\section{CONCLUSION}

We have presented a framework to generate sequences of $2 \mathrm{D}$ and 3D LLSM images depicting astrocytic activity. To the best of our knowledge, this is the first simulation tool of its kind. Our framework is based on a kinetic model, and the spatio-temporal diversity of calcium signals is simulated inside real astrocyte processes. The variety of our synthetic datasets realistically represents the complexity of experimental data. We believe that it will be helpful to develop computerized learning-based methods to analyze astrocytic calcium signals in 3D+time images.

\section{ACKNOWLEDGMENTS}

There are no relevant financial or non-financial interests to disclose.
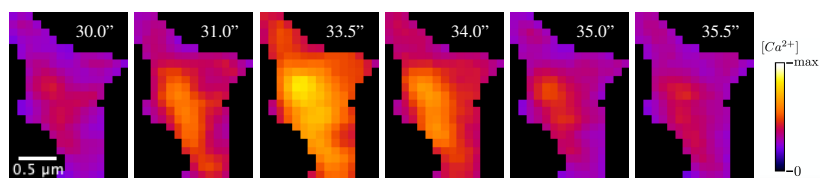

(a) Calcium puff.
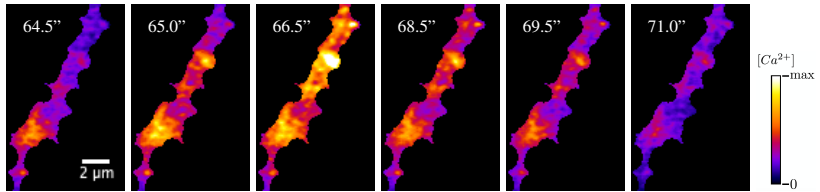

(b) Calcium growing wave.
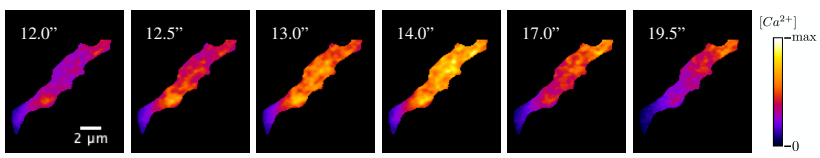

(c) Calcium moving wave.

Fig. 4. Maximum intensity projections of calcium puff (a) and waves (b)-(c) from real 3D+time LLSM images $(\Delta t=0.5 \mathrm{~s})$. Voxelsize: $0.1025 \times 0.1025 \times 0.1066 \mu \mathrm{m}^{3}$. Source: M. Arizono, M. Ducros, U. V. Nägerl, Interdisciplinary Institute for Neuroscience, Bordeaux.
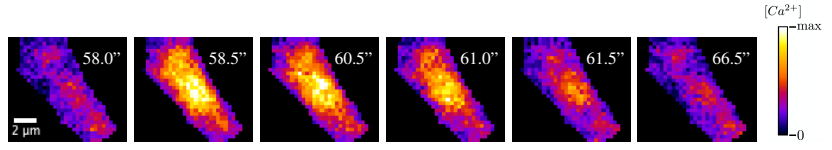

(a) Calcium puff.
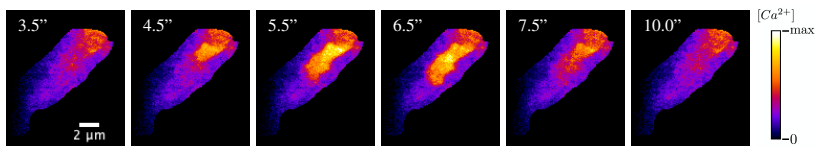

(b) Calcium growing wave.
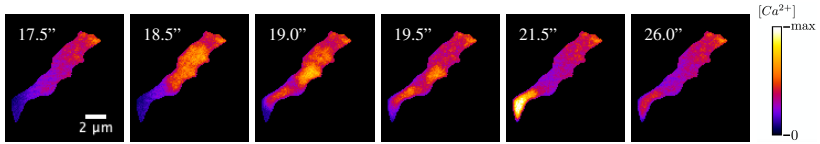

(c) Calcium moving wave.

Fig. 5. Maximum intensity projections of calcium puff (a) and waves (b)-(c) from our simulated 3D+time LLSM images $(\Delta t=0.5 \mathrm{~s})$.
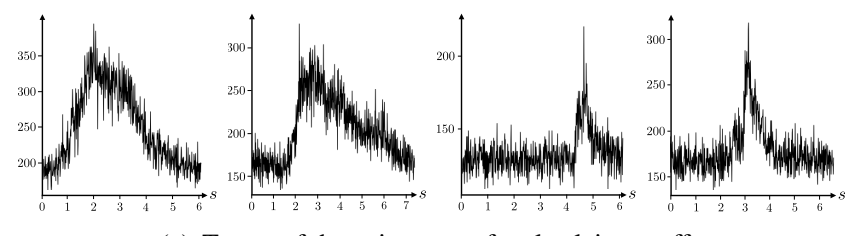

(a) Traces of the epicenters of real calcium puffs.
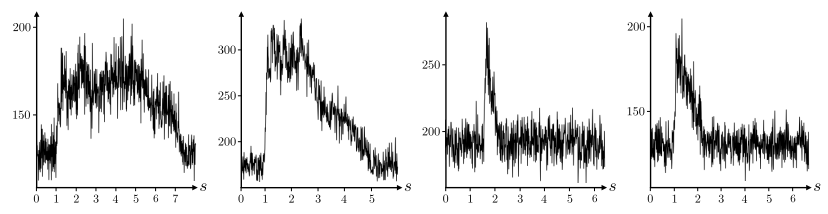

(b) Traces of the epicenters of simulated calcium puffs.

Fig. 6. Traces of the epicenters of calcium puffs present in real (a) and synthetic (b) 2D+time LLSM images $(\Delta t=0.01 \mathrm{~s})$. Source of the real data: M. Arizono, M. Ducros, U. V. Nägerl, Interdisciplinary Institute for Neuroscience, Bordeaux. 


\section{COMPLIANCE WITH ETHICAL STANDARS}

Experimental procedures were in accordance with the French National Code of Ethics on Animal Experimentation and approved by the Committee of Ethics of Bordeaux. All procedures were in accordance with the guidelines of the European Directive 2010/63/UE.

\section{REFERENCES}

[1] M. Santello, N. Toni, and A. Volterra, "Astrocyte function from information processing to cognition and cognitive impairment," Nat. Neurosci., vol. 22, no. 2, pp. 154-166, Jan. 2019.

[2] A. Araque, G. Carmignoto, P. G. Haydon, S. H. R. Oliet, R. Robitaille, and A. Volterra, "Gliotransmitters travel in time and space,” Neuron, vol. 81, no. 4, pp. 728-739, Feb. 2014.

[3] R. Srinivasan, B. S. Huang, S. Venugopal, A. D. Johnston, H. Chai, H. Zeng, P. Golshani, and B. S. Khakh, " $\mathrm{Ca}^{2+}$ signaling in astrocytes from IP3R $2^{-/-}$mice in brain slices and during startle responses in vivo," Nat. Neurosci., vol. 18, no. 5, pp. 708-717, Apr. 2015.

[4] E. Bindocci, I. Savtchouk, N. Liaudet, D. Becker, G. Carriero, and A. Volterra, "Three-dimensional $\mathrm{Ca}^{2+}$ imaging advances understanding of astrocyte biology," Science, vol. 356, no. 6339, May 2017.

[5] A. Semyanov, C. Henneberger, and A. Agarwal, "Making sense of astrocytic calcium signals - from acquisition to interpretation," Nat. Rev. Neurosci., pp. 551-564, Sept. 2020.

[6] A. Reichenbach, A. Derouiche, and F. Kirchhoff, "Morphology and dynamics of perisynaptic glia," Brain Res. Rev., vol. 63, no. 1-2, pp. 11-25, Feb. 2010

[7] M. Arizono, V. V. G. K. Inavalli, A. Panatier, T. Pfeiffer, J. Angibaud, F. Levet, M. J. T. Ter Veer, J. Stobart, L. Bellocchio, K. Mikoshiba, G. Marsicano, B. Weber, S. H. R. Oliet, and U. V. Nägerl, "Structural basis of astrocytic $\mathrm{Ca}^{2+}$ signals at tripartite synapses," Nat. Commun., vol. 11, no. 1, pp. 1-15, Apr. 2020.

[8] T. Manninen, R. Havela, and M.-L. Linne, "Computational models for calcium-mediated astrocyte functions," Front. in Comp. Neurosci., vol. 12, pp. 14, Apr. 2018.

[9] A. Denizot, H. Berry, and S. Venugopal, Intracellular Calcium Signals in Astrocytes, Computational Modeling of, pp. 1-12, Springer New York, March 2020.

[10] B.-C. Chen, W. R. Legant, K. Wang, L. Shao, D. E. Milkie, M. W. Davidson, C. Janetopoulos, X. S. Wu, J. A. Hammer, Z. Liu, et al., "Lattice light-sheet microscopy: imaging molecules to embryos at high spatiotemporal resolution," Science, vol. 346, no. 6208, Oct. 2014.

[11] M. Ducros, A. Getz, M. Arizono, V. Pecoraro, M. FernandezMonreal, M. Letellier, U. V. Nägerl, and D. Choquet, "Lattice light sheet microscopy and photo-stimulation in brain slices," in Neural Imaging and Sensing 2019, San Francisco, California, Feb. 2-7 2019, vol. 10865, p. 1086508.

[12] S. Berlin, E. C. Carroll, Z. L. Newman, H. O. Okada, C. M. Quinn, B. Kallman, N. C. Rockwell, S. S. Martin, J. C. Lagarias, and E. Y. Isacoff, "Photoactivatable genetically encoded calcium indicators for targeted neuronal imaging," Nat. Methods, vol. 12, no. 9, pp. 852-858, July 2015.
[13] S. Venugopal, R. Srinivasan, and B. S. Khakh, "GECIquant: Semi-automated detection and quantification of astrocyte intracellular $\mathrm{Ca}^{2+}$ signals monitored with GCaMP6f," in Comp. Gliosci., pp. 455-470. Jan. 2019.

[14] A. Agarwal, P.-H. Wu, E. G. Hughes, M. Fukaya, M. A. Tischfield, A. J. Langseth, D. Wirtz, and D. E. Bergles, "Transient opening of the mitochondrial permeability transition pore induces microdomain calcium transients in astrocyte processes," Neuron, vol. 93, no. 3, pp. 587-605, Feb. 2017.

[15] Y. Wang, G. Shi, D. J. Miller, Y. Wang, C. Wang, G. Broussard, Y. Wang, L. Tian, and G. Yu, "Automated functional analysis of astrocytes from chronic time-lapse calcium imaging data," Front. in Neuroinform., vol. 11, pp. 48, July 2017.

[16] Y. Wang, N. V. DelRosso, T. V. Vaidyanathan, M. K. Cahill, M. E. Reitman, S. Pittolo, X. Mi, G. Yu, and K. E. Poskanzer, "Accurate quantification of astrocyte and neurotransmitter fluorescence dynamics for single-cell and population-level physiology," Nat. Neurosci., vol. 22, no. 11, pp. 1936-1944, 2019.

[17] J. Tremblay, A. Prakash, D. Acuna, M. Brophy, V. Jampani, C. Anil, T. To, E. Cameracci, S. Boochoon, and S. Birchfield, "Training deep networks with synthetic data: Bridging the reality gap by domain randomization," in Proc. of the IEEE Conf. on Comp. Vis. Pattern Recognit. Workshops, Salt Lake City, Utah, US, June 18-22 2018, pp. 969-977.

[18] A. Denizot, M. Arizono, U. V. Nägerl, H. Soula, and H. Berry, "Simulation of calcium signaling in fine astrocytic processes: Effect of spatial properties on spontaneous activity," PLoS Comp. Bio., vol. 15, no. 8, pp. e1006795, Aug. 2019.

[19] M. W. Sherwood, M. Arizono, C. Hisatsune, H. Bannai, E. Ebisui, J. L. Sherwood, A. Panatier, S. H. R. Oliet, and K. Mikoshiba, "Astrocytic $\mathrm{IP}_{3}$ Rs: Contribution to $\mathrm{Ca}^{2+}$ signalling and hippocampal LTP," Glia, vol. 65, no. 3, pp. 502513, Jan. 2017.

[20] A. P. Thillaiappan, N. B.and Chavda, S. C. Tovey, D. L. Prole, and C. W. Taylor, " $\mathrm{Ca}^{2+}$ signals initiate at immobile $\mathrm{IP}_{3}$ receptors adjacent to ER-plasma membrane junctions," Nat. commun., vol. 8, no. 1, pp. 1-16, Nov. 2017.

[21] I. Bezprozvanny, J. Watras, and B. E. Ehrlich, "Bell-shaped calcium-response curves of $\operatorname{lns}(1,4,5) \mathrm{P}_{3}$ - and calcium-gated channels from endoplasmic reticulum of cerebellum," Nature, vol. 351, no. 6329, pp. 751-754, June 1991.

[22] S. Swillens, G. Dupont, L. Combettes, and P. Champeil, "From calcium blips to calcium puffs: theoretical analysis of the requirements for interchannel communication," Proc. of the Natl. Acad. of Sci., vol. 96, no. 24, pp. 13750-13755, Nov. 1999.

[23] G. W. De Young and J. Keizer, "A single-pool inositol 1, 4, 5trisphosphate-receptor-based model for agonist-stimulated oscillations in $\mathrm{Ca}^{2+}$ concentration," Proc. of the Natl. Acad. of Sci., vol. 89, no. 20, pp. 9895-9899, Oct. 1992.

[24] A. F. Frangi, W. J. Niessen, K. L. Vincken, and M. A. Viergever, "Multiscale vessel enhancement filtering," in $M I C$ CAI, Heidelberg, Berlin, 1998, pp. 130-137.

[25] P. Perona and J. Malik, "Scale-space and edge detection using anisotropic diffusion," Trans. on Patt. Anal. and Mach. Intell., vol. 12, no. 7, pp. 629-639, July 1990.

[26] J. Schindelin, I. Arganda-Carreras, E. Frise, V. Kaynig, M. Longair, et al., "Fiji: An open-source platform for biological-image analysis," Nat. Methods, vol. 9, no. 7, pp. 676-682, June 2012. 